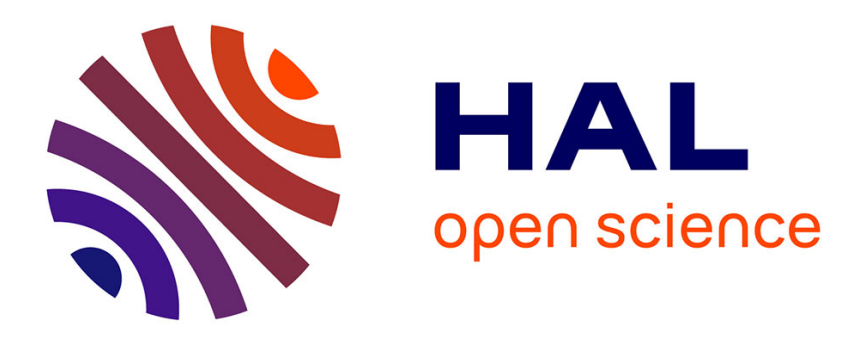

\title{
CPG-based Controllers can Trigger the Emergence of Social Synchrony in Human-Robot Interactions
}

\author{
Melanie Jouaiti, Patrick Henaff
}

\section{To cite this version:}

Melanie Jouaiti, Patrick Henaff. CPG-based Controllers can Trigger the Emergence of Social Synchrony in Human-Robot Interactions. ARSO 2018 - IEEE International Workshop on Advanced Robotics and its Social Impacts, Sep 2018, Genoa, Italy. hal-01825464v2

\section{HAL Id: hal-01825464 \\ https://hal.science/hal-01825464v2}

Submitted on 18 Apr 2019

HAL is a multi-disciplinary open access archive for the deposit and dissemination of scientific research documents, whether they are published or not. The documents may come from teaching and research institutions in France or abroad, or from public or private research centers.
L'archive ouverte pluridisciplinaire HAL, est destinée au dépôt et à la diffusion de documents scientifiques de niveau recherche, publiés ou non, émanant des établissements d'enseignement et de recherche français ou étrangers, des laboratoires publics ou privés. 


\title{
CPG-based Controllers can Trigger the Emergence of Social Synchrony in Human-Robot Interactions
}

\author{
Melanie Jouaiti ${ }^{1}$ and Patrick Henaff ${ }^{2}$
}

\begin{abstract}
Synchronization is an indissociable part of social interactions between humans, especially in gestural communication. With the emergence of social robotics and assistance robots, it becomes paramount for robots to be socially accepted and for humans to be able to connect with them. As a consequence, synchronization mechanisms should be inherent to any robot controllers, allowing the adaption to the interacting partner in any rhythmic way necessary. In this paper, plastic Central Pattern Generators (CPG) have been implemented in the joints of the robot Pepper that has to learn to wave back at a human partner. Results show that the CPG-based controller leads to adaptive waving synchronized with the human partner, thus proving that the CPG-based controller can achieve synchronization.
\end{abstract}

\section{INTRODUCTION}

With the increasing popularity of social robots and the prospect of them being more and more pervasive, it becomes essential for robots to be accepted. Social acceptance would indeed allow robots to effectively perform their tasks at welcome desks, at home, in hospitals, schools, train stations... It has become quite obvious that an appealing appearance and smooth complex gestures would not be enough to achieve this integration. Robots still lack a paramount ability. Humans require their interaction partner to behave in an appropriate way in order to connect, that is, a socially acceptable response in accord with the social and human context is expected. Consequently, robots need to be able to synchronize and hence, their controllers have to incorporate mechanisms capable of triggering the synchronization. One way of achieving this consists in using Central Pattern Generators (CPGs) endowed with plasticity mechanisms, allowing them to alter their intrinsic properties in conjunction with proprioceptive or exteroceptive information.

The waving gesture and its social implications, to this day and to our knowledge, have never been studied. Though not directly concerned with waving, [?] introduced a model designed to imitate rhythmic arm movements with the NAO robot. But this model is not able to learn to synchronize with an external signal. Oscillators with close frequency are merely chosen in a reservoir and while the frequency can be slightly entrained during the interaction, the oscillators go back to their original properties right afterwards. Nevertheless, waving is an important part of the gestural communication between humans and although it presents with great

\footnotetext{
*This work was supported by CPER 2015-2020, plateform IT2MPSCIARAT, region Nancy Grand-Est, France

${ }^{1}$ Melanie Jouaiti is with Université de Lorraine, CNRS, LORIA, F-54000 Nancy, France melanie.jouaiti@loria.fr

${ }^{2}$ Patrick Henaff with Université de Lorraine, CNRS, LORIA, F-54000 Nancy, France patrick.henaffeloria.fr
}

variety (one, two joints, hand-only waving), its rhythmic nature can be linked to other studies on rhythmic gestures and leads us to assume that it induces the same locking mechanisms observed in other rhythmic social interactions [?]. It is thus postulated that the waving gesture, though trivial in appearance is actually a complex communication act, triggering synchronization phenomena and that its study would be an interesting step in understanding human gestural communication.

In this paper, plastic CPGs, i.e. CPGs which incorporate plastic and synaptic Hebbian plasticity, were implemented in the joints of the Pepper robot in order to perform synchronized waving with a human partner. Results show that our controller triggers the emergence of motor coordination between the robot and the human partner waving and hence of social synchrony.

In section II, interpersonal synchrony and synchrony measures are introduced. Section III details the model of sensorimotor loop employed. Then in section IV, the results of the synchrony analysis are explained. Finally, section V concludes this paper and mentions future prospects.

\section{SYNCHRONY IN INTERPERSONAL RELATIONSHIPS}

Though the terms synchrony, synchronization and coordination are often interchangeably employed, their meanings actually differ. Synchrony denotes the state of two or more events occurring at the same time. Coordination describes events occurring with a constant phase difference (which can differ from zero). Synchronization indicates that the events are in phase (phase difference is zero) or in anti-phase (phase difference is $\pi$ ), it can be restricted to in-phase in some studies. Synchrony is ubiquitous in complex systems, such as biological systems, ranging from traffic regulation [?] to animal behavioral patterns [?].

Interpersonal synchrony can be observed in many different situations: head movements or posture in a conversation [?], [?], crowd behavior [?], team sports. For humans, physical and social interactions induce gestural and verbal/non-verbal communication based on rhythmic mechanisms and rhythmic movements. People, in rhythmic social interactions, experience two phenomena which can be observed in oscillators: the magnet effect which entrains both systems until they are coupled and synchronized; the maintenance effect which is the struggle of each system to conserve its own intrinsic frequency [?]. These mechanisms could play a fundamental role in physical and social interpersonal interactions [?], [?] and could be an emergent feature of these interactions 
where humans adapt to each other and learn from each interaction, generating synchronization phenomena and creating conscious or unconscious links between people [?]. Scientists assume that emotional and social interactions involve a coupling between individuals which is achieved thanks to neural structures with similar properties as those implicated in the neural control of movements. In the case of inter-limb coordination within a single individual, neural synchrony has been observed between cortical areas of the brain [?] and also between two distinct brains in the case of coordination of oscillatory motions between two individuals [?], [?].

\section{A. Measures of synchrony}

Interpersonal coordination can be captured by video analysis, motion tracking or psychophysiological and neurophysiological methods (See [?] for a review). Afterwards, several measures can be employed to analyze the data and quantify the degree of synchronization.

Firstly, different correlation or coherence indexes are available. They provide only one averaging value to evaluate the whole interaction. These indexes cause a lot of information loss. Indeed, with a single value for the whole interaction, information such as synchronization length, beginning of synchronization or loss of synchronization are not available. Besides, generalized Partial Directed Coherence is suitable for multivariate autoregressive models and has been used to evaluate synchronization in neural signals [?] and breathing rhythms [?]. The intersubject correlation analysis is mostly used to analyze fMRI data [?]. Meanwhile, cross-recurrence quantification analysis detects intricate recurrent structuring between paired signals [?], [?], [?]. It is a nonlinear method that allows quantification of dynamical systems and their trajectories because corresponding signals are non-stationary. Contrary to most methods, it does not perform any averaging which usually leads to data loss. Furthermore, several different methods first compute the instantaneous phase with the Hilbert transform or with wavelet transform. Next, the phases are compared by computing the mean vector length of the angular dispersions of the phase difference in a complex space [?] or by simply comparing the instantaneous phase of two signals [?], [?] or by multiplying the wavelet transforms of both systems [?].

In this paper, two synchrony measures were selected for the study of waving signals: the instantaneous Phase Locking Value and the synchrosqueezing transform.

1) Phase Locking Value (PLV): The Phase Locking Value (PLV) has been introduced by [?] to measure coordination in brain signals. It relies on the assumption that both signals are locked with a constant phase difference but the PLV allows for deviations and evaluates this spread. It ranges from 0 (no coordination) to 1 (perfect coordination). First, the Hilbert transform is computed, providing the instantaneous phase $\phi$ for each signal, then the instantaneous PLV can be obtained:

$$
P L V(t)=\frac{1}{N}\left|\sum_{i=0}^{N} e^{j\left(\phi_{1}(i)-\phi_{2}(i)\right)}\right|
$$

with $N$ the sliding window size, $j=\sqrt{-1}, \phi_{k}$ the instantaneous phase of signal $k$.

2) Synchrosqueezing: Synchrosqueezing introduced in the context of analyzing auditory signals [?] aims to decompose signals into constituent components with time-varying oscillatory characteristics. It is assumed that signals have following form:

$$
f(t)=\sum_{k=1}^{K} f_{k}(t)+e(t)
$$

with $f_{k}(t)=A_{k}(t) \cos \left(2 \pi \phi_{k}(t)\right)$ a Fourier-like oscillatory mode and $e(t)$ noise.

First, the continous Wavelet transform is computed:

$$
W_{s}(a, b)=\int s(t) a^{-1 / 2} \overline{\psi\left(-\frac{t-b}{a}\right)} d t
$$

with $\psi$ an appropriate mother wavelet. A candidate instantaneous frequency $\omega_{s}(a, b)$ for the signal s can be derived:

$$
\omega_{s}(a, b)=-i\left(W_{s}(a, b)\right)^{-1} \frac{\delta W_{s}(a, b)}{\delta b}
$$

The Wavelet Synchrosqueezing transform of $f$ is defined as:

$$
T_{s}\left(\omega_{l}, b\right)=(\Delta \omega)^{-1} \sum_{a:\left|\omega_{s}(a, b)-\omega_{l}\right| \leq \Delta \omega / 2} W_{s}(a, b) a^{-3 / 2} \Delta a
$$

with $\omega_{l}$ the center frequency bin where frequencies are closer to $\omega_{l}$ than to any other frequency.

\section{Cross Synchrosqueezing}

Synchrosqueezing provides frequency information for one single signal but synchronization concerns two signals, so let us define Cross Synchrosqueezing as the product of Wavelet Synchrosqueezing transform obtained for each signal. This operation, similar to the cross wavelet transform [?] is designed to highlight regions were the frequency is similar for both signals and remove the other frequencies.

\section{MODEL OF SENSORI-MOTOR LOOP}

The optical flow of a hand waving in front of the Pepper camera is the input of the first CPG. Since two joints waving is realized, the output of the first CPG is used as the input for the second CPG (see Figure ??). The output of the CPG is thus considered an articular position here (the position control mode to command the joints is employed). The output of each CPG is mapped to the acceptable range of values for the appropriate joint.

\section{A. The CPG-based architecture}

Several models of CPGs have been proposed over the years in order to understand human and animal motor control, mainly aiming at locomotion control in robotics [?], [?], [?]. CPGs are neuronal structures located in the spinal cord and able to generate rhythmic and discrete activities that can be initiated, modulated and reset by different kinds of signals (afferent or efferent). Different levels of CPG modeling exist 


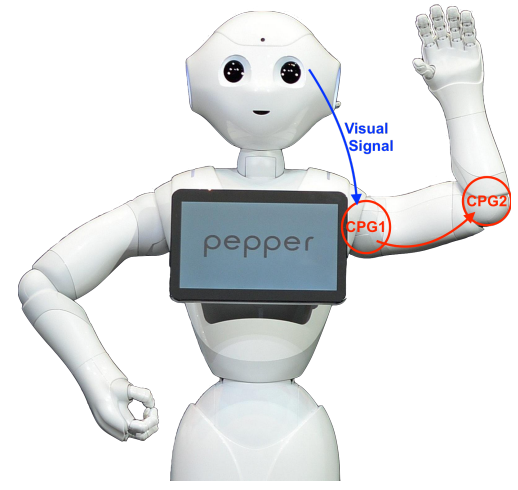

Figure 1. Experiment setup with the robot Pepper. The optical flow detected is fed as an input to first CPG, while the input of the second CPG is the output of the first.

from the microscopic level that takes into account many details in the biophysical operation of the neurons like the famous Hodgkin-Huxley model [?], to the macroscopic level that tries to reproduce the functionality of a population of neurons using non-linear oscillators like Van der Pol [?], [?], Rayleigh [?] or Hopf [?], [?].

Using non-linear oscillator models (also called relaxationoscillators) for oscillating neurons in CPGs is particularly interesting because they have the property of natural synchronization with an external signal, provided the frequency of this signal is not too different from the oscillator's own intrinsic frequency [?]. Thus, non-linear oscillators are suitable models to explain and reproduce the synchrony phenomena that emerge in interpersonal coordination, especially if they are implemented at the rhythmic level of a CPG. In this case, by acting like a dynamic attractor, they facilitate the selfsynchronization of the CPG with the dynamic of the limb controlled by the CPG.

An interesting bio-inspired model of half-center CPG for mammal locomotion was proposed by McCrea and Rybak [?]. The CPG is divided into two parts representing the extensor and flexor muscles and has four layers: Rhythm Generator, Pattern Formation, Sensory Neurons and Motoneurons. It also takes sensory feedback into account.

For the rhythm generator neurons, Rowat-Selverston cells are used. They are generalized versions of the Van der Pol oscillator and can exhibit the four characteristic behaviors of a biological rhythmic neuron, i.e. endogenous bursting, plateau potential, post-inhibitory rebound and quiescence.

In this paper, we introduce many equations without much explanation, consequently we recommend that the reader read [?], where the CPG model is extensively detailed, for a full understanding. Following equations are used for the rhythmic cells:

$$
\begin{aligned}
\dot{V}_{i_{\{E, F\}}} & =y_{i_{\{E, F\}}}-W \frac{y_{i_{\{E, F\}}}}{1+e^{-4 y_{i_{\{F, E\}}}}}+\epsilon_{i_{\{E, F\}}} F_{i} \\
\dot{y}_{i_{\{E, F\}}} & = \\
& \left(\sigma_{f}-\frac{\tau_{m}}{\tau_{s}}-1-\sigma_{f} \tanh ^{2}\left(\frac{\sigma_{f}}{A_{f_{i}}} V_{i_{\{E, F\}}}\right)\right) \frac{y_{i_{\{E, F\}}}}{\tau_{m}} \\
& -\frac{1+\sigma_{s}}{\tau_{s} \tau_{m}} V_{i_{\{E, F\}}}+\frac{A_{f_{i_{\{E, F\}}}}}{\tau_{s} \tau_{m}} \tanh \left(\frac{\sigma_{f} V_{i_{\{E, F\}}}}{A_{f_{i_{\{E, F\}}}}}\right)
\end{aligned}
$$

With $i \in \mathbb{N}$, designating the joint id. $F_{i}$ is the input of the $\mathrm{CPG}, \epsilon$ a synaptic weight designed to scale the input and the term in $\mathrm{W}$ models the mutual inhibition between the extensor and flexor rhythmic cells. $\mathrm{V}$ is the membrane potential and $\tau_{m}$ and $\tau_{s}$ are time constants, $A_{f}$ influences the output amplitude, while $\sigma_{f}$ determines whether the neuron is able to oscillate or not. $\sigma_{s}$ is a gain. For more details, refer to [?].

Pattern Formation neuron PF, Sensory neuron SN and Motoneuron $\mathrm{MN}$ are defined as follows [?]:

$$
\begin{array}{r}
P F\left(V_{i_{\{E, F\}}}\right)=P F_{i_{\{E, F\}}}=\frac{1}{1+e^{\frac{-V_{i}\{E, F\}}{2}}} \\
S N_{s}\left(v_{m e s_{i}}\right)=S N_{i, s}=\frac{1}{1+e^{\alpha_{s} p o s_{i_{m e s}}}} \\
M N\left(P F_{i_{\{E, F\}}}, S N_{i, s}\right)=\frac{1}{1+e^{\alpha_{m}\left(P F_{i_{\{E, F\}}}-S N_{i, s}\right)}}
\end{array}
$$

with $\alpha_{s}=-0.061342$ and $\alpha_{m}=3$. pos $_{\text {mes }_{i}}$ is the angular position measured for the given joint.

\section{B. The plasticity mechanisms}

Several plasticity mechanisms inspired by [?] are integrated to the $\mathrm{CPG}$, allowing it to alter its intrinsic properties to adapt to the input. How the plasticity rules were derived won't be detailed here, since it is not the subject of this paper but details can be found in [?]. There are merely provided to the reader for exhaustiveness.

Frequency learning is defined as:

$$
\begin{aligned}
& \dot{\sigma}_{s_{i_{\{E, F\}}}}= \\
& 2 \epsilon F_{i} \sqrt{\tau_{m} \tau_{s}\left(1+\sigma_{s_{i_{\{E, F\}}}}-\sigma_{f}\right)} \frac{y_{i_{\{E, F\}}}}{\sqrt{V_{i_{\{E, F\}}}^{2}+y_{i_{\{E, F\}}}^{2}}}
\end{aligned}
$$

The learning rule for the amplitude $A_{f}$ is the following:

$$
\dot{A}_{f_{i_{\{E, F\}}}}=-\mu\left(\left(\frac{\nu \sigma_{f} V_{i_{\{E, F\}}}}{A_{f_{i_{\{E, F\}}}}}\right)^{2}-F_{i}^{2}\right)
$$

The adaptation of the synaptic weight $\epsilon$ is realized with:

$$
\dot{\epsilon}_{i_{\{E, F\}}}=\lambda \tanh (\xi|F|)\left(1-\left(\epsilon_{i_{\{E, F\}}} F_{i}\right)^{2}\right)
$$

$\mu$ and $\lambda$ are learning steps. $\nu$ and $\xi$ are scaling factors. 


\section{RESULTS OF THE EXPERIMENTS}

\section{A. Protocol}

Ten waving interactions were performed by the same human with the Pepper robot (Softbanks Robotics). Each interaction lasts $50 \mathrm{~s}$. Between $\mathrm{t}=0$ and $\mathrm{t}=20 \mathrm{~s}$, the human waves slowly at approximately $0.5 \mathrm{~Hz}$, then between $\mathrm{t}=20 \mathrm{~s}$ and $t=40 \mathrm{~s}$, the waving frequency increases at $1 \mathrm{~Hz}$. Finally, between $\mathrm{t}=40 \mathrm{~s}$ and $\mathrm{t}=50 \mathrm{~s}$, no waving occurs. The Pepper camera provides pictures at $20 \mathrm{fps}$. The optical flow of the hand waving was obtained with a background subtraction algorithm, followed by simple image processing steps, all of which implemented in OpenCV version 3.4. Furthermore, the signal was passed through a low-pass filter with a $5 \mathrm{~Hz}$ cutoff frequency in order to remove some detection aberrations. Waving was performed with two joints: the shoulder roll joint and the elbow yaw joint. The input of the shoulder CPG is the optical flow detected. The shoulder CPG output is the input of the elbow CPG. The controller is implemented in Python 2.7 and runs with ROS. Coordination across the waving series was evaluated with the previously introduced PLV and synchrosqueezing.

\section{B. Emergence of Synchrony}

On Figure ??, the optical flow detected and angular positions of the shoulder and elbow joints for a single interaction are represented as an interaction example. The emergence of coordination can be observed after a transitory phase, every time the input varies: at the beginning of the interaction and at $\mathrm{t}=20 \mathrm{~s}$ when the waving frequency increases. It can also be noted that while the shoulder joint is in phase with the optical flow the whole interaction, the elbow joint is in anti-phase during the slow waving phase and in-phase during the fast waving phase. Note that while $1 \mathrm{~Hz}$ can hardly be considered as fast, it was imposed by the camera frame-rate and robot bandwidth.

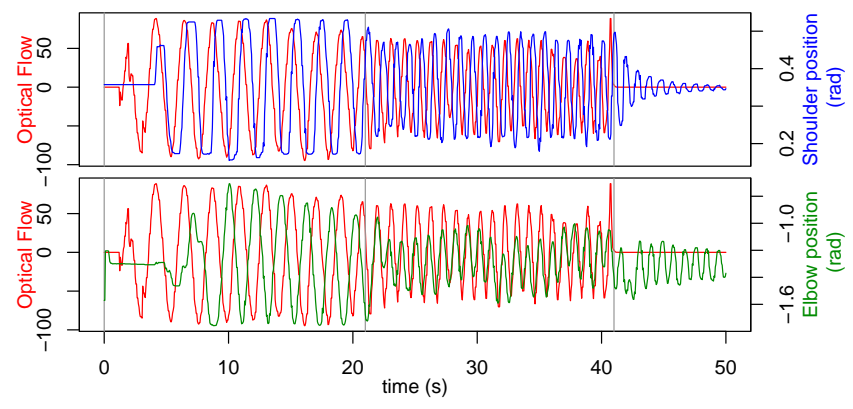

Figure 2. Top, in red, optical flow and in blue, angular position of the shoulder joint; Bottom, in red, optical flow and in blue, angular position of elbow joint. The vertical lines represent the beginning of the interaction, the frequency change and the end of waving.

The PLV is used as an error measurement metric and was implemented using a moving window size $N=50$ (see equation ??).
Figure ?? represents the superimposed PLV for the ten interactions. It can be plainly observed that though the shoulder joint quickly coordinates with the optical flow (t $=7 \mathrm{~s}$ ), the elbow joint experiences a longer transition phase before achieving coordination $(\mathrm{t}=9.7 \mathrm{~s})$. Similarly, at $\mathrm{t}=$ $20 \mathrm{~s}$, when the waving frequency changes, the transition is more distinct for the elbow joint than for the shoulder joint. Waving in the higher frequency appears less stable and with more variability than for the lower frequency, especially for the elbow joint.

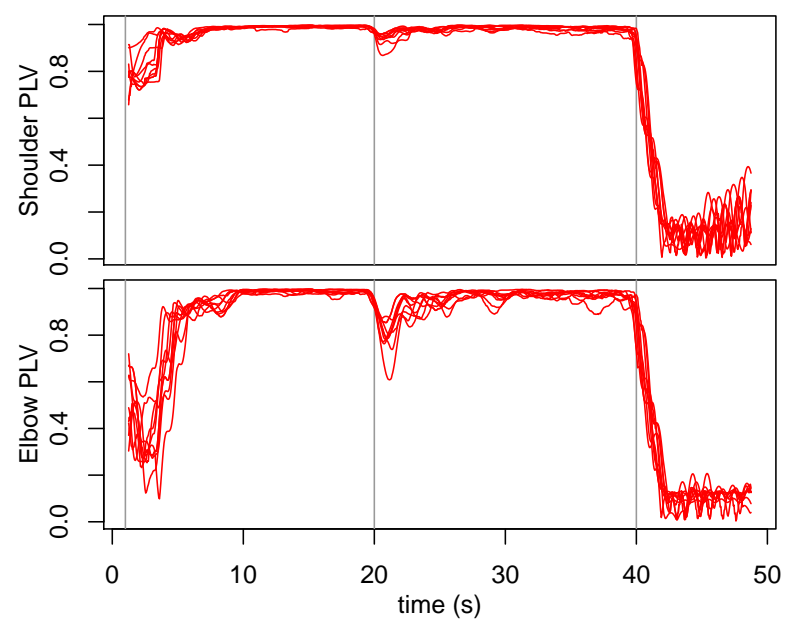

Figure 3. Top, PLV for the shoulder joint and the optical flow; Bottom, PLV for the elbow joint and the optical flow. The PLV for ten interactions are superimposed.

For the synchrosqueezing analysis, the Matlab toolbox implementation by [?], [?] was employed.

Figure ?? represents the ten cross synchrosqueezing transforms superimposed. The color represents the power of the frequency specter: the darker the blue, the stronger the specter. It can be observed that in the transition periods (beginning, frequency change, end), the specter is larger and fainter than during the phases where the signals are coordinated.

It should also be noticed that the strength of cross synchrosqueezing for the elbow joint appears strong only around $\mathrm{t}=8.5 \mathrm{~s}$, after the shoulder joint $(\mathrm{t}=6.0 \mathrm{~s})$. The same phenomenon can also be observed at the frequency change. This confirms what has already been observed for the PLV: the elbow joint achieves coordination after the shoulder joint.

\section{Repeatability of synchrony}

Despite sub-optimal initial conditions and human variability evidenced by the lack of overlap in the synchrosqueezing curves (Figure ??), our controller is able to achieve coordination every time, as proven by the PLV curves displaying a great overlap (Figure ??). Thus, the coordination observed previously is not merely due to chance but the controller indeed triggers the emergence of coordination (the PLV tends to reach 1.0) after a transitory phase where the system adapts to the new input information. 

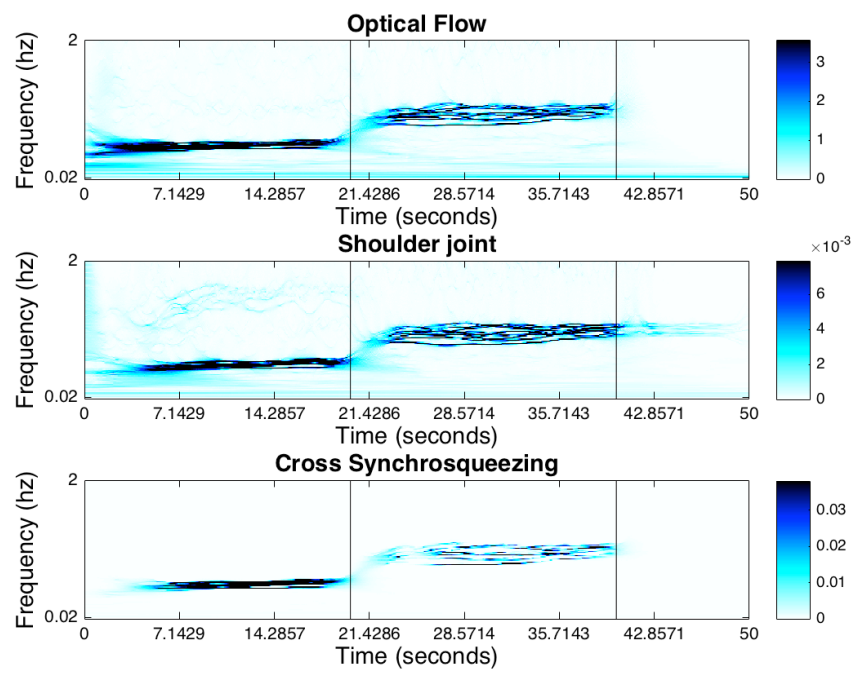

Figure 4. Left for the shoulder joint, right for the elbow joint. Top, optical flow synchrosqueezing; Middle, joint synchrosqueezing; Bottom, cross synchrosqueezing. The synchrosqueezing for ten interactions are superimposed.

\section{CONCLUSION}

In this paper, we showed that CPG-based controllers endowed with plasticity mechanisms are able to synchronize with an external visual signal. This synchronization property enables motor synchronization which in turn, triggers the emergence of social synchronization between the robot and the human. This was achieved by analyzing the synchronization between the optical flow and articular command values across ten different interactions, thanks to the PLV and synchrosqueezing transform. Despite the human variability obviously present in different interactions, results show an interesting coherence with signals often overlapping. Besides, one advantage of using biological inspiration, consists in not having to compute nor use a dynamical model of the robot.

It has been constantly observed that the elbow joint synchronizes after the shoulder joint. This is perfectly coherent since the input of elbow joint is the output of the shoulder joint, as such, it cannot possibly synchronize before the shoulder joint does. The elbow joint undergoes some sort of exploratory phase before achieving synchronization. It may be interesting to investigate the influence and relevance of other inputs for the second CPG. Ongoing experiments with another robot already allow us to study the influence of torque feedback.

Moreover, it was observed that the robot's response could switch from anti-phase to in-phase for the elbow joint when the waving frequency increased. The CPG naturally generates a signal which is in anti-phase with the input signal, this can be clearly observed for slow waving. However, for fast waving, we are confronted to bandwidth limitations and the joints do not answer as they should. While the command is still in anti-phase, the robot answer undergoes a phase shift. While this phenomenon [?], [?], [?] is widely observed in human rhythmic movements and modeled by the HKB model [?], in our case it is merely due to the robot's inability to perform such rapid movements. It was indeed observed that the elbow joint response was most unsatisfactory for frequencies above $0.5 \mathrm{~Hz}$. If we wish robots to be able to act in a human-like way and synchronize with a human partner, a hardware improvement for actuators (compliance and higher bandwidth) would be required, so that such low frequency limitations do not arise.

In future works, we will study synchronization in the waving gesture further by equipping the human partner with motion sensors.

\section{ACKNOWLEDGMENT}

We thank Shinji Omori for his implementation of the PLV. We wish to thank Ing. Olivier Rochel and Dorian Goepp for their help in solving technical difficulties with Pepper. We also thank Dr. Alain Dutech and Francis Colas for their help with ROS. 